\title{
The Effect of Cognitive-Behavioral Stress Management Group Training on Anxiety, Depression, Stress, and Re-admission in Asthma Patients in Tehran Ziaiyan Hospital
}

Fatemeh Ahmadi ${ }^{1}$, Masoud Fallahi-Khoshknab ${ }^{2,}{ }^{*}$, Abolfazl Rahgoi ${ }^{3}$, Farahnaz MohammadiShahboulaghi ${ }^{4}$, Pouria Rezasoltani ${ }^{5}$

${ }^{1}$ M.Sc. Student of Mental Health Nursing, Department of Nursing, Faculty of Rehabilitation Sciences, University of Social Welfare and Rehabilitation Sciences, Tehran, Iran

2 Professor of Nursing Department of Nursing, School of Rehabilitation Sciences Research Center in Emergency and Disaster Health University of Social Welfare and Rehabilitation Sciences, Tehran, Iran

${ }^{3}$ Instructor of Nursing Department of Nursing, School of Rehabilitation Sciences University of Social Welfare and Rehabilitation Sciences, Tehran, Iran

${ }^{4}$ Professor of Nursing Department of Nursing, School of Rehabilitation Sciences Research Center on Aging University of Social Welfare and Rehabilitation Sciences, Tehran, Iran

${ }^{5}$ Department of Biostatics, University of Social Welfare and Rehabilitation Sciences, Tehran, Iran

* Corresponding author: Masoud Fallahi-Khoshknab, Professor of Nursing Department of Nursing, School of Rehabilitation Sciences Research Center in Emergency and Disaster Health University of Social Welfare and Rehabilitation Sciences, Tehran, Iran. E-mail: msflir@yahoo.com

How to Cite this Article:

Ahmadi F, Fallahi-Khoshknab M, Rahgoi A, Mohammadi-Shahboulaghi F, Rezasoltani P. The Effect of Cognitive-Behavioral Stress Management Group Training on Anxiety, Depression, Stress, and Re-admission in Asthma Patients in Tehran Ziaiyan Hospital. J North Khorasan Univ Med Sci. 2020;7(1):19-29.

DOI: $10.29252 /$ ijrn-06403

\begin{tabular}{l}
\hline Received: 30 Oct 2019 \\
Accepted: 27 Apr 2020 \\
\hline Keywords: \\
Anxiety, Stress, Depression, \\
Asthma, Cognitive-Behavioral, \\
Stress Management \\
\hline C) 2020 Iranian Journal of \\
Rehabilitation Research in Nursing
\end{tabular}

\begin{abstract}
Introduction: Psychological therapies can play an essential role in the improvement of anxiety, depression, and stress in patients with asthma. The purpose of this study was to investigate the effectiveness of cognitive-behavioral stress management group therapy in group therapy on anxiety, depression, stress, and re-admission in asthmatic patients.

Methods: In this quasi-experimental study, 38 patients with asthma were selected and randomly assigned to intervention and control groups. DASS-21 questionnaire was used for data collection, which was completed at pre-test, post-test, and follow-up. Cognitive-behavioral stress management therapy was administered to the intervention group on ten sessions, eight but no intervention conducted for the control group. Data were analyzed using ANCOVA, oneway ANOVA, t-test, and repeated measures.

Results: The results showed that there was a significant difference between the mean score of depression in the intervention group before $(6.42 \pm 7.9)$ and after the intervention $(5.05 \pm 5.55)$ $(P=0.00)$. There was also a significant difference between the mean score of depression between the response $(5.05 \pm 5.55)$ and control groups $(13.47 \pm 9.49)$ in the first month $(P=$ 0.017). There was a significant difference between the mean scores of stress before $(10.74 \pm$ $5.97)$ and after $(9.05 \pm 5.22)$ the intervention in the intervention group $(P=0.00)$. The mean score of the anxiety variable after the response was a significant difference between the intervention group $(4.53 \pm 5.37)$ and control groups $(10.95 \pm 7.90)(P=0.01)$. There was no significant difference in re-admission in the intervention and control groups before and after the intervention.

Conclusions: The results of the present study indicate the effectiveness of cognitive-behavioral stress management therapy in improving anxiety, depression, and stress in patients with asthma. Also, results showed the high level of anxiety, depression and stress in patients with asthma and the effectiveness of cognitive-behavioral stress management training intervention and the
\end{abstract} need for serious attention to psychological therapies in this group of patients. 


\section{Extended Abstract}

\section{OBJECTIVE}

A

$\mathrm{s}$ a significant health issue in most parts of the world, asthma is difficult to diagnose and treat. Many people with this disease die each year (1, $2)$. A study has shown that the prevalence of asthma symptoms in Iran is $9 \%$ in adults and $10 \%$ in children and adolescents (5). Asthma is the third leading cause of hospitalization of children (6). According to the results from a study done by Ptiras et al. (2009) an investigation of the level of anxiety and depression among patients with asthma and Chronic obstructive pulmonary disease (COPD), there is a direct relationship between anxiety/depression and asthma (9). Researches emphasize that psychological factors such as anxiety, depression, and stress are considered triggers for asthma. In this regard, they have been investigating psychological therapies for the signs and symptoms of asthma. It has been reported that psychological interventions, including such as cognitive-behavioral therapy stress management (15), have a positive effect on the treatment of patients with asthma. In various studies, the effectiveness of CBT-based stress management for people with chronic diseases and normal people has been studied, and useful results have been obtained. Norte et al. (2011) showed in research that CBT-based stress management interventions are very effective in increasing resiliency and decreasing anxiety and depression (18). Findings of Emami and et al. (2017) showed that CBT-based stress management interventions increase the resiliency and hope of women with cancer in the experimental group (19). Sepehrianazar and Tabizadeh (2017) demonstrated in their study that the level of resiliency and rumination showed a significant difference between experimental and control groups after CBT-based stress management interventions (20). The results of a study by Charmchi et al. (2016) also showed that CBT-based stress management could reduce anxiety and increase the resiliency of postmenopausal women (21). These studies have shown that training of CBT-based stress management has had a significant effect on reducing stress, anxiety, and depression among chronic patients.

\section{MATERIALS AND METHODS}

The study population of this quasi-experimental study included all patients with asthma referred to the internal ward of Ziaeyan Hospital in Tehran in 2019. The total number of samples determined using the following formula and information from similar studies was 19 in each group (22), and the probability of Type 1 error and power of the test were, respectively, $(\alpha) 0.05$ and (1- $\beta=$
0.8). Data gathering was done using a questionnaire that had two parts. The first part consisted of demographic information, including personal information (e.g., age, gender, level of education, marital status, the number of hospitalization, etc.). The second part consisted of the DASS-21 questionnaire applied by Lovibond (23). In the study of Moradi-Panah (2005), Cronbach's alpha coefficients in the same questionnaire for depression, anxiety, and stress were $94 \%, 92 \%$, and $82 \%$, respectively (26). The researcher got written informed consent letters from the participants and started gathering data using the questionnaire. The researcher then began to perform the intervention for the intervention group using the Stress Management Training Kit based on cognitive-behavioral techniques, and no psychological intervention was performed for the control group during the study period. The training intervention was performed in the form of a group for ten weekly sessions, and each session lasted 2 hours for the patients of the intervention group. One month after the intervention, the questionnaires were re-completed by the participants and the number of previous hospitalizations due to asthma was recorded based on the remarks of the patients and medical records. Then, in the follow-ups performed after the intervention, two months after the end of the intervention and three months after the end of the intervention, the questionnaires and the number of hospitalization sessions for the patients of intervention group and control group within three months after the intervention were completed and recorded. Analyzing data from the study was performed using 25-SPSS software and the analysis of covariance tests (ANCOVA) repeated-measures and ANOVA. To conduct this study, ethical principles in medical research on Helsinki human subjects were used (27).

\section{RESULTS}

The results showed that there was a significant difference between the mean score of depression in the intervention group before $(6.42 \pm 7.9)$ and after the intervention $(5.05 \pm 5.55)(\mathrm{P}=0.00)$. There was also a significant difference between the mean score of depression between the response $(5.05 \pm 5.55)$ and control groups $(13.47 \pm 9.49)$ in the first month $(\mathrm{P}=$ $0.017)$. There was a significant difference between the mean scores of stress before $(10.74 \pm 5.97)$ and after $(9.05 \pm 5.22)$ the intervention in the intervention group $(\mathrm{P}=0.00)$. The mean score of the anxiety variable after the intervention was a significant difference between the intervention group $(4.53 \pm 5.37)$ and control groups 
$(10.95 \pm 7.90)(\mathrm{P}=0.01)$. There was no significant difference in re-admission in the intervention and control groups before and after the intervention.

Table 1. Mean and Standard Deviation of the Mean Stress Score after the Intervention in Different Times of Measurements for Intervention and Control Groups

\begin{tabular}{|c|c|c|c|c|c|c|}
\hline \multirow[t]{2}{*}{ Variable } & \multicolumn{2}{|c|}{ The First Month (After The Intervention) } & \multicolumn{2}{|c|}{ The Second Month (Follow-Up) } & \multicolumn{2}{|c|}{ The Third Month (Follow-Up) } \\
\hline & Mean & Standard Deviation & Mean & Standard Deviation & Mean & Standard Deviation \\
\hline \multicolumn{7}{|l|}{ Stress } \\
\hline Intervention & 9.05 & 5.22 & 7.79 & 4.94 & 7.58 & 4.60 \\
\hline Control & 16.42 & 8.83 & 17.26 & 7.87 & 17.47 & 9.42 \\
\hline \multicolumn{7}{|l|}{ Anxiety } \\
\hline Intervention & 4.53 & 5.37 & 4.21 & 5.33 & 4.11 & 4.69 \\
\hline Control & 10.95 & 7.90 & 11.16 & 7.95 & 10.74 & 8.54 \\
\hline \multicolumn{7}{|l|}{ Depression } \\
\hline Intervention & 5.05 & 5.55 & 4.21 & 5.37 & 3.89 & 4.59 \\
\hline Control & 13.47 & 9.49 & 12.63 & 8.77 & 12.32 & 8.67 \\
\hline
\end{tabular}

\section{CONCLUSION}

The results of the present study indicate the effectiveness of cognitive-behavioral stress management therapy in improving anxiety, depression, and stress in patients with asthma. Data showed a high level of anxiety, depression, and stress in patients with asthma and the effectiveness of cognitive-behavioral stress management training intervention and the need for serious attention to psychological therapies in this group of patients.

The results of the present study showed that there was no significant relationship between hospitalization before and after the intervention. The cohort study of Singh et al. (2016) demonstrated that the number of admission during one month was higher among patients with chronic lung obstruction with psychological disorders such as anxiety and depression than those with no psychology disorders, which is inconsistent with our study (37).

\section{Ethical Considerations}

In this study, we received ethical code from the Research Council of Rehabilitation Sciences and Social Welfare University with some (IR.USWR.REC.007).

\section{Funding or Supports}

Research Council of Rehabilitation Sciences and Social Welfare University.

\section{Author's Contribution}

Fatemeh Ahmadi and Masoud Fallahi-Khoshknab developed the original idea, and the protocol, abstracted, wrote the manuscript and is guarantor. Abolfazl Rahgoi and Farahnaz MohammadiShahboulaghi contributed to the development of the contract, abstracted data, and prepared the manuscript; Pouria RezaSoltani contributed the analyzed data.

\section{Conflict of Interest}

In this article, there was no reported any conflict of interest.

\section{Applicable Remarks}

Because cognitive-behavioral stress management training has been shown to reduce anxiety, depression, and stress in patients with asthma, nurses, hospitals, and health care providers can use exercise to manage stress. Single-person and group play an important role in promoting the health of patients with asthma and patients with other chronic diseases. Therefore, managers and planners of hospitals can include stress management training in in-service training programs for nurses and doctors and reduce the burden of patients in hospitals and health centers to increase the quality of life of patients with chronic diseases, which is a critical factor. It is undeniable that they should take steps to improve the health of the community.

\section{Acknowledgments}

The authors gratefully acknowledge the Research Council of Rehabilitation Sciences and Social Welfare University (Grant Number: 708) for financial support. 


\title{
تأثير آموزش كروهى مديريت استرس به شيوه شناختى - رفتارى بر اضطراب، افسردىى، استر و بسترى مجدد بيماران مبتلابه آسم
}

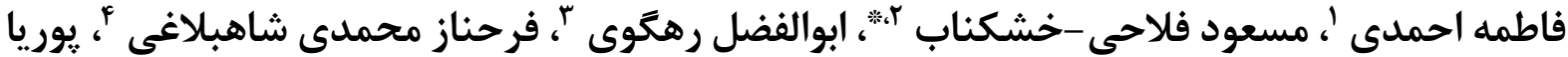 \\ رضا سطانى ها
}

'دانشجوى كارشناسى ارشد يرستارى بهداشت روان، كروه يرستارى، دانشكده علوم توانبخشى، دانشكاه علوم بهزيستى و توانبخى، تهران،

r استاد آموزش يرستارى، كروه برستارى، دانشكده علوم توانبخى، مركز تحقيقات سلامت در حوادث و بلايا، دانشكاه علوم بجزيستى و توانبخشى، تهران، ايران

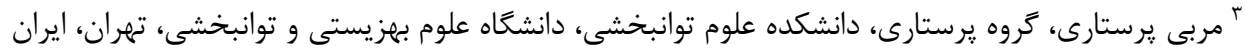

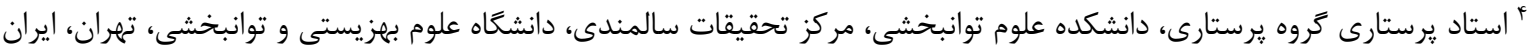

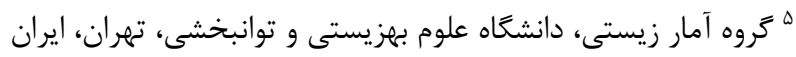

" نويسنده مسئول: مسعود فلاحى-خشكناب، گروه يرستارى، دانشكده علوم توانبشى، مركز تحقيقات سلامت در حوادث و بلايا، دانشكاه علوم بهزيستى و توانبخشى، تهران، ايران. ايميل: msflir@yahoo.com

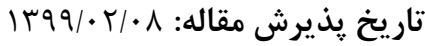

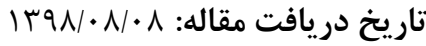

مقدمه: بيماران مبتلا به آسم درجات بالايى از استرس را تجربه مىنمايند. آموزشهاى روان شناختى مىتوانند سهم بسزايى در بهبود اضطراب، افسردكى

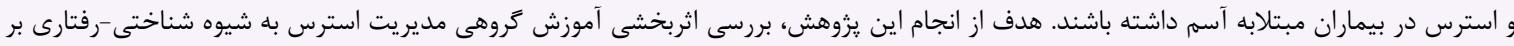
اضطراب، افسردگى و استرس بيماران مبتلابه آسم بود.

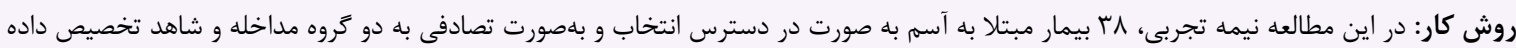

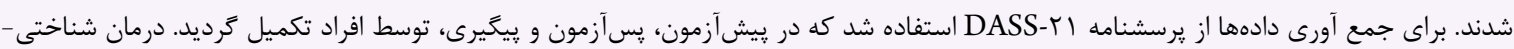

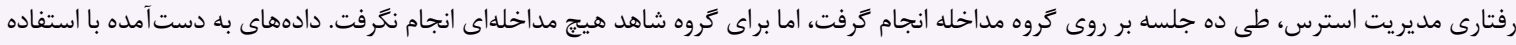

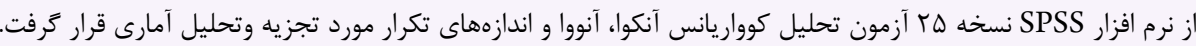

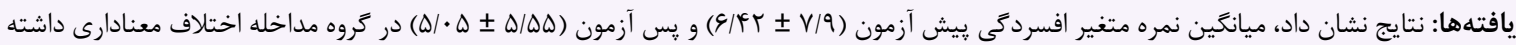

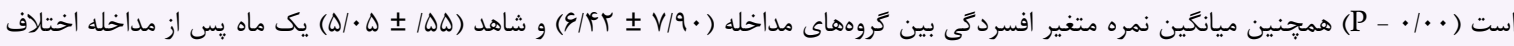

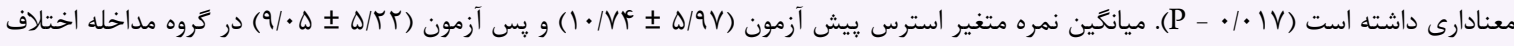

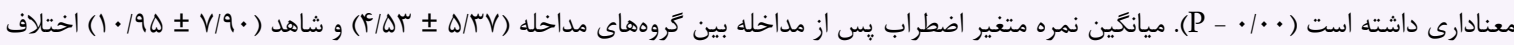

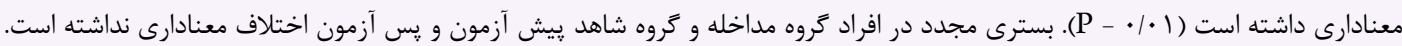

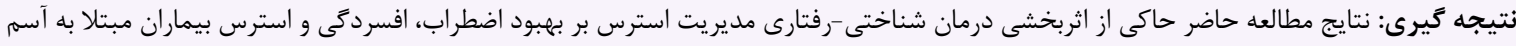

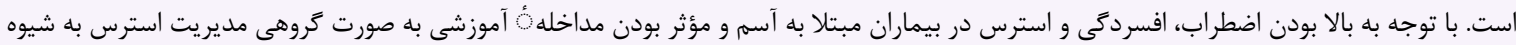

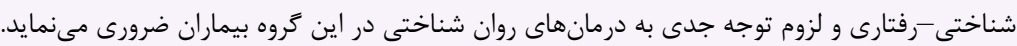

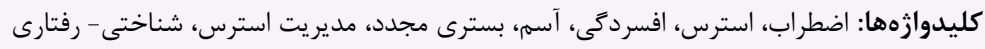


روشهاى رفتارى مثل (آرام سازى، مراقبه، حساسيتزدايى منظهم) و يا

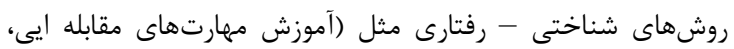

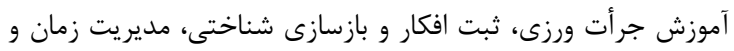

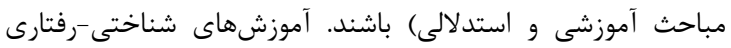

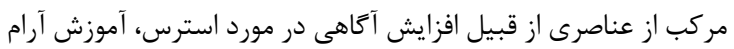

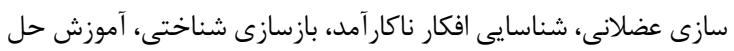

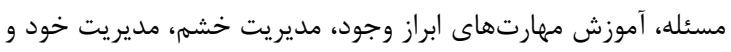
برنامه ريزى فعاليتها مىباشد [IV]

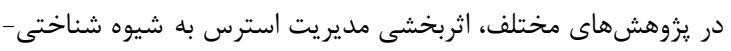

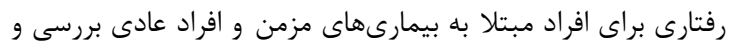

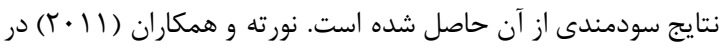

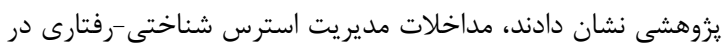

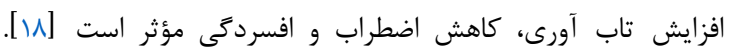

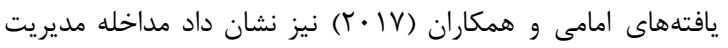

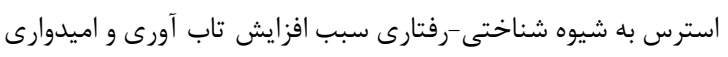

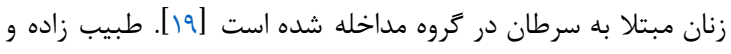

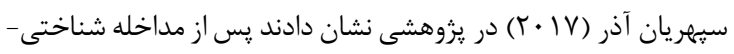

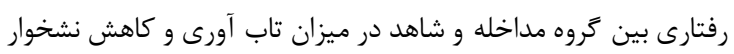

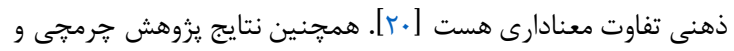

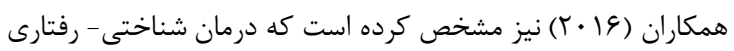

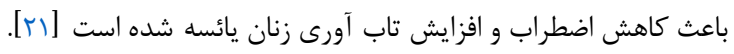

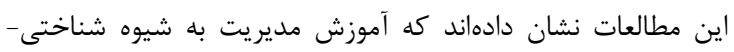

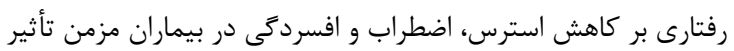
بسزايى داشته است. با توجه به مطالب فوق درمىيابيم كه بيماران مبتلا به آسم مستعد

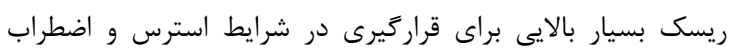

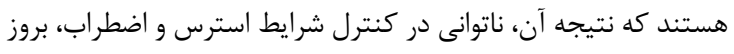

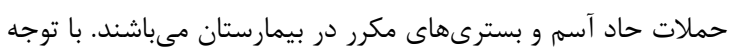

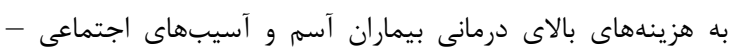

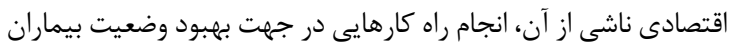

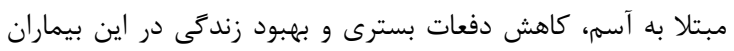
عملى بسيار هوشمندانه و ضرورى است. اين مطالعه باهدف تعيين تأثير

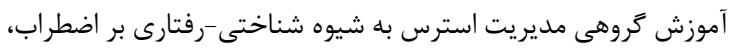

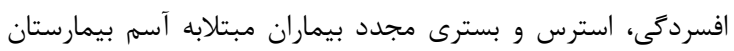

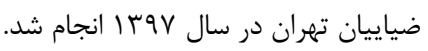

\section{روش كار}

اين مطالعه بلهورت نيمه تجربى بود و جامعه مورد يزوهش شامل

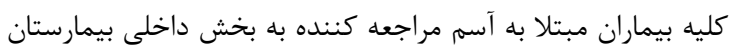

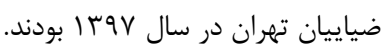

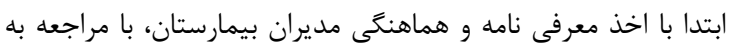
بخش داخلى بيمارستان ضياييان تهران، اقدام به نمونه كيرى افراني

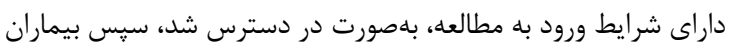

آسم (Asthma) يك مشكل عمده بهداشتى در اغلب نقاط دنيا است

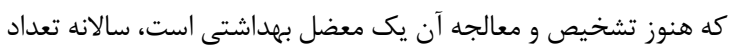

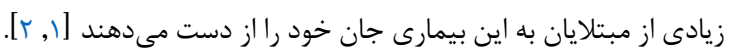

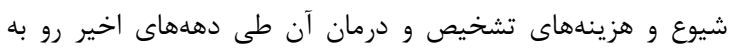

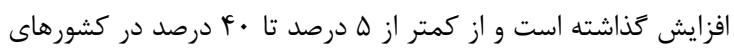

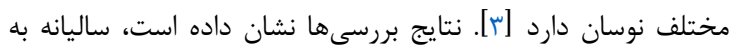

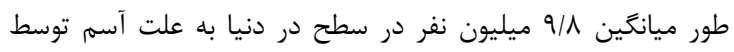

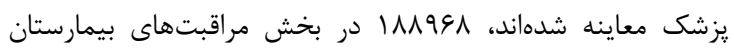

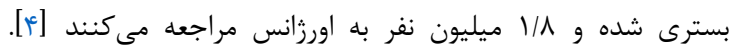

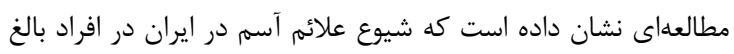

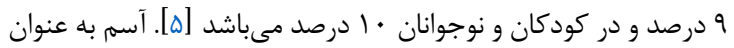

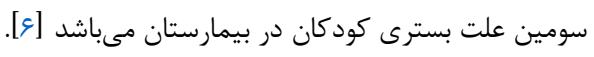

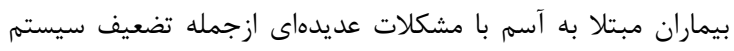

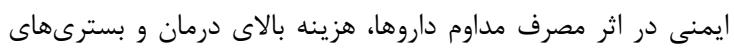

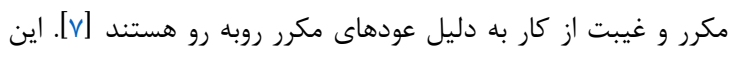

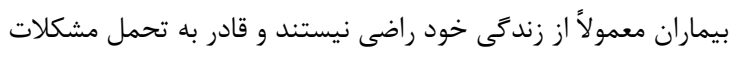

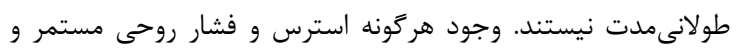

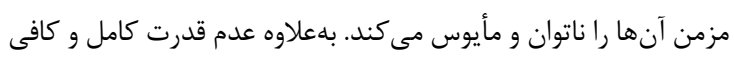

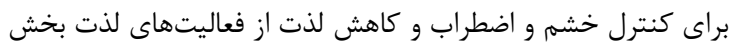

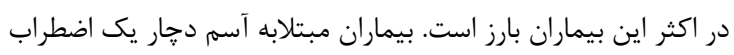

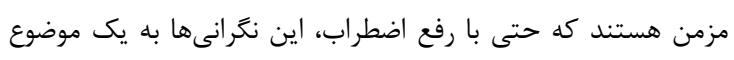
ديخر انتقال مىيابد [م].

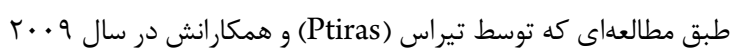

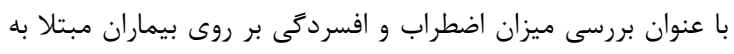

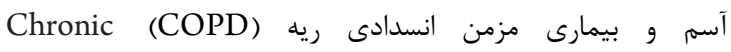
Obstructive Pulmonary Disease كه ارتباط مستقيم بين اضطراب و افسردگى و تننى نفس وجود دارد

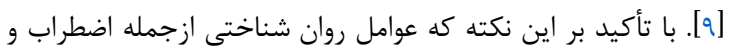

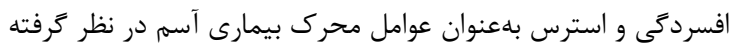

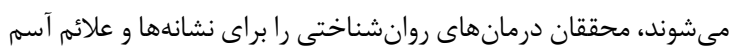

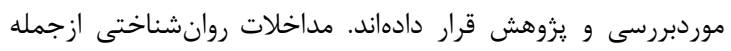

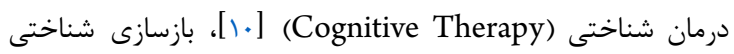

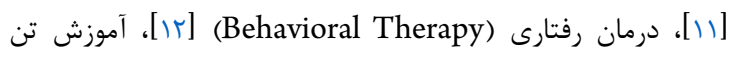
آرامى (Relaxation Therapy)، بيوفيدبك (Biofeedback) [بان،

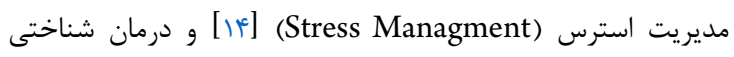
رفتارى مديريت استرس (Cognitive \& Behavioral Therapy) [10]، تأثير مثبتى را در بهببود اين بيماران كزارش كردهاند. مديريت

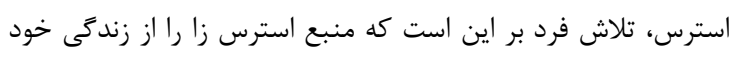

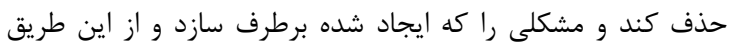
تعادل و آرامش خود را به دست آورد [عاد].

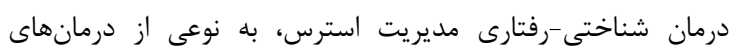

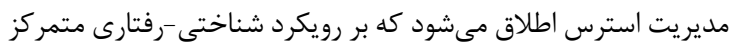

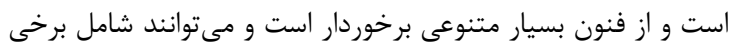


تعداد نمونه مورد مطالعه با استفاده از فرمول زير و اطلاعات حاصل از

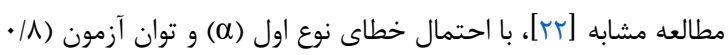

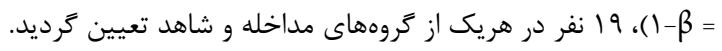

موردمطالعه، با استفاده از روش تصادفى ساده به دو كروه مداخله و شاهد تخصيص داده شدند.

$$
n=\frac{2 \sigma_{d}^{2}\left(z_{1-\frac{\alpha}{2}}+z_{1-\beta}\right)^{2}}{\delta^{2}}=\frac{2 \times 177.7 \times(1.96+0.84)^{2}}{12.25^{2}} \approx 19
$$

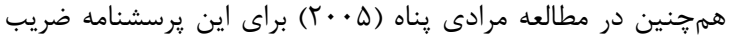

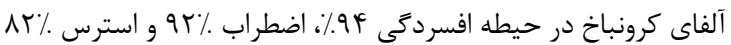

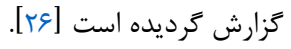

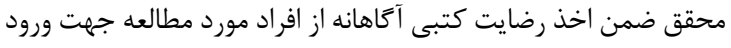

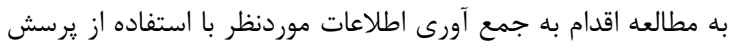

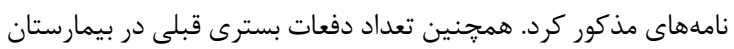

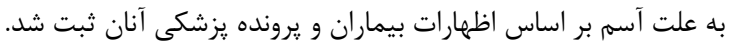

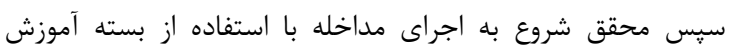

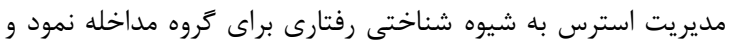

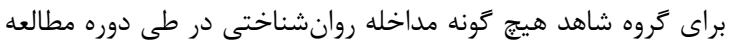

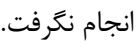

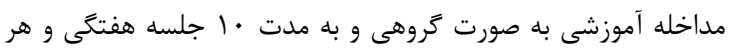

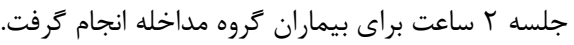

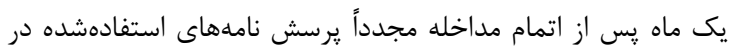

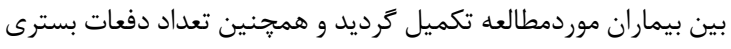

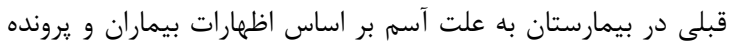

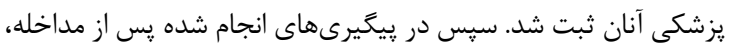

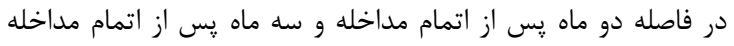

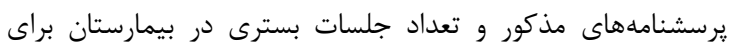

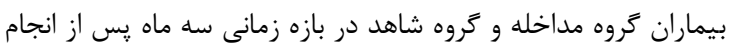
مداخله تكميل و ثبت كر ديد.

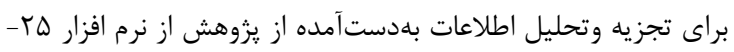

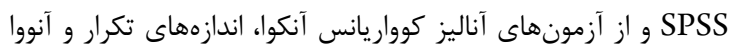

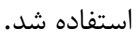

\section{يافتهها}

در اين مطالعه ^ץ فرد مبتلا به آسم مورد مطالعه قرار كَرفتند، 19 نفر

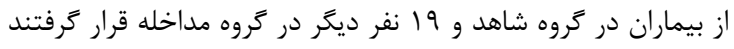

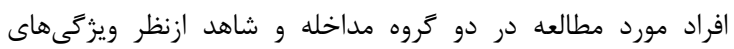

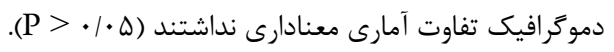

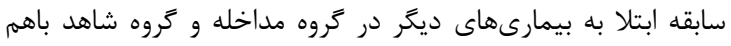

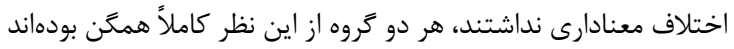

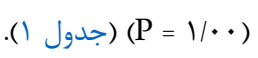

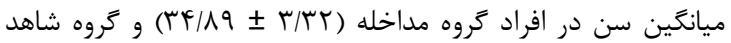

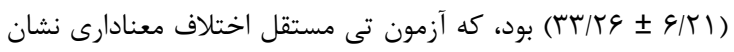

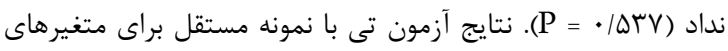

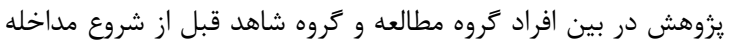

$$
\text { در جدول ب بنشان داده است. }
$$

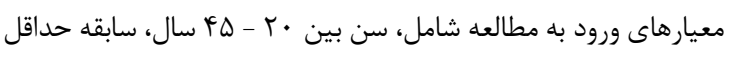

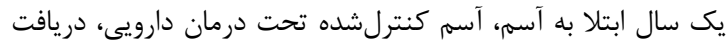

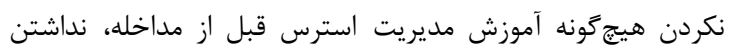

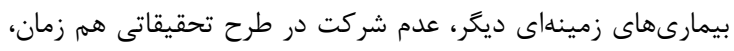

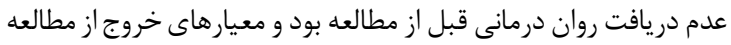

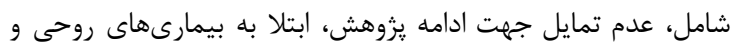

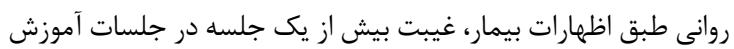

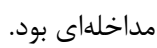

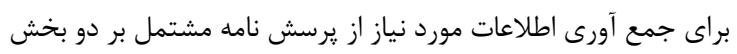

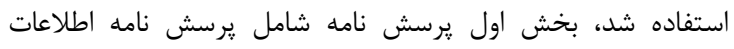

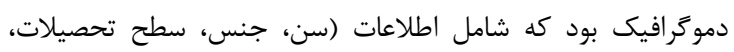

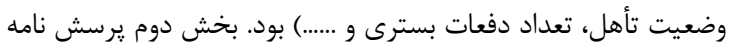

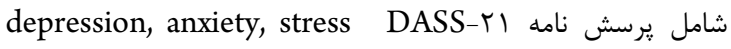

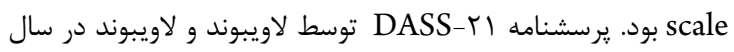

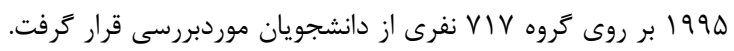

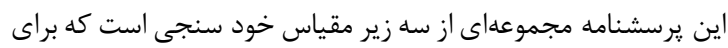

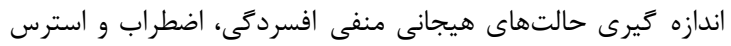

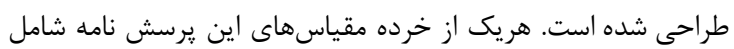

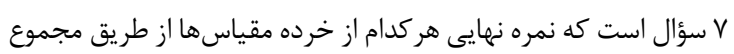

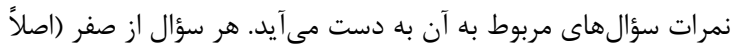

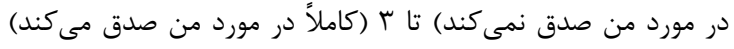

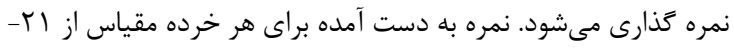

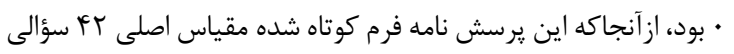

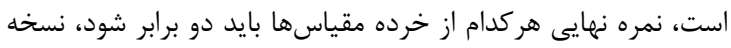

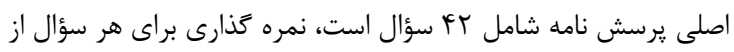

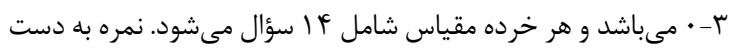

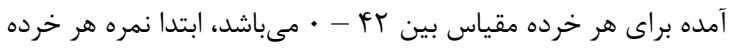

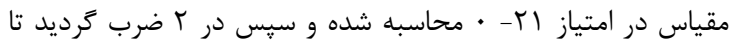

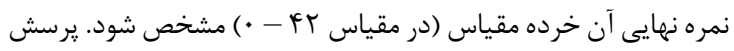

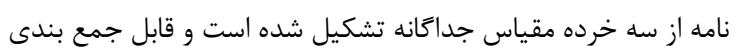
نمىباشد [سب].

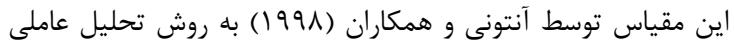

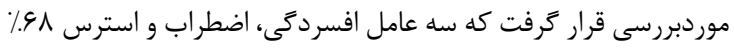

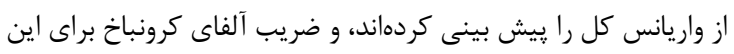

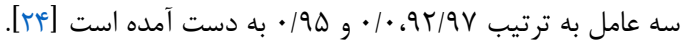

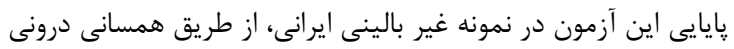

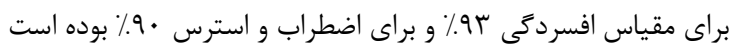

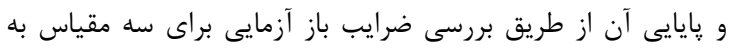

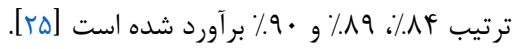




\begin{tabular}{|c|c|c|c|c|}
\hline \multicolumn{2}{|c|}{ آزمون كاى مربع } & \multicolumn{2}{|c|}{ فراوانى } & \multirow{2}{*}{ 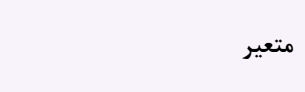 } \\
\hline سطح معنادارى & آماره كاى مربع & 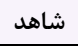 & 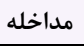 & \\
\hline \multirow[t]{3}{*}{$\cdot / 1 \vee 9$} & $1 / 11$ & & & جنسيت \\
\hline & & 9 & $\Delta$ & 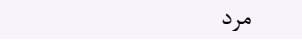 \\
\hline & & 1. & 19 & زن \\
\hline \multirow[t]{3}{*}{$\cdot / \Delta 9 \Delta$} & $1 / \cdot 4 \cdot$ & & & وضعيت تأهل \\
\hline & & $v$ & 9 & مجرد ومطلقه \\
\hline & & ir & ir & متأهل \\
\hline \multirow[t]{4}{*}{.$/ .49$} & N/TAK & & & سطح تحصيلات \\
\hline & & ir & $\Delta$ & زيردييلم ودييلم \\
\hline & & 4 & 9 & فوق دييلم \\
\hline & & 1 & $\wedge$ & كارشناسى و كارشناسى ارشد \\
\hline \multirow[t]{3}{*}{$1 / \cdot \cdot$} & $\cdot 1 \cdots$ & & & سابقه ابتلا \\
\hline & & 9 & 4 & بله \\
\hline & & ir & ir & خير \\
\hline
\end{tabular}

جدول r. يافتهاى آزمون تى با نمونه مستقل براى متغيرهاى يُوهش قبل از شروع مداخله در كروههاى مداخله و شاهد

\begin{tabular}{|c|c|c|c|}
\hline $\mathbf{P}$ & انحراف معيار & ميانكين & متغير \\
\hline \multirow[t]{3}{*}{$\cdot|\Delta V|$} & & & افسردحى قبل از مداخله \\
\hline & $V / 9$. & S/4T & مداخله \\
\hline & $9 / \Delta 9$ & $\mid r / T V$ & شاهد \\
\hline \multirow[t]{3}{*}{ • /TAT } & & & اضطراب قبل از مداخله \\
\hline & 9191 & G/FT & مداخله \\
\hline & V/DT & $9 / 19$ & شاهد \\
\hline \multirow[t]{3}{*}{. Rtr } & & & استرس قبل از مداخله \\
\hline & $\Delta / 9 V$ & $1 \cdot / V^{F}$ & مداخله \\
\hline & $\Lambda / \Lambda$. & 19111 & شاهد \\
\hline
\end{tabular}

ماه اول، دوم و سوم) اختلاف معنادارى نداشت (P= • P (PAF). اما

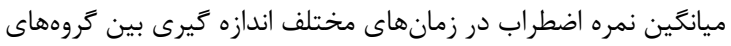

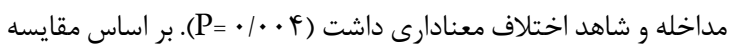

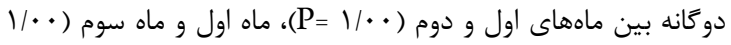
P=

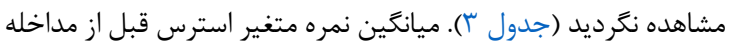

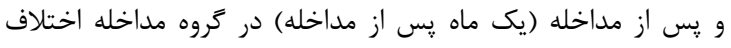

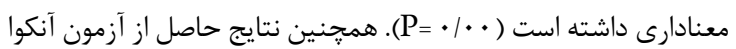

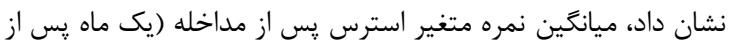

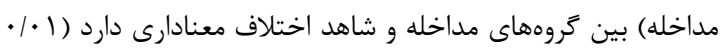

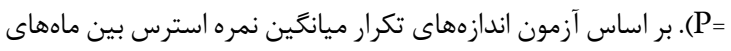

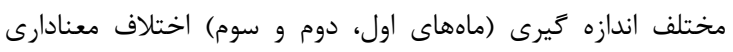

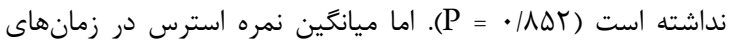

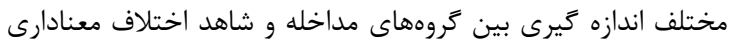

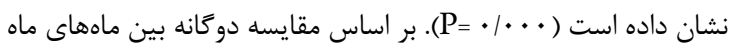

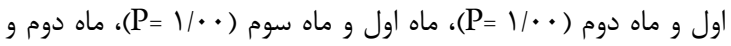

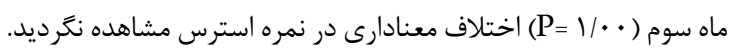
ميانگين نمره استرس در افراد گروه مداخله در ماههاى يس از مدرد مداخله به ترتيب كاهش نشان داده است (جدول س).
ميانَّين نمره متغير افسردَى قبل از مداخله و يس از مداخله (يك ماه

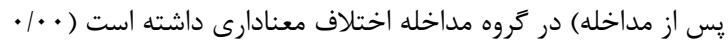
P=

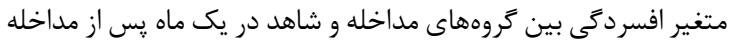

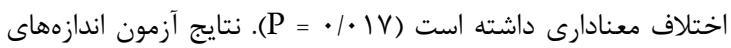

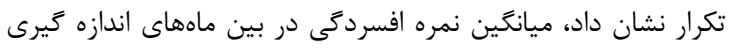

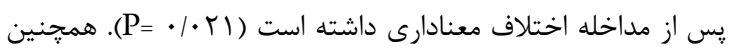

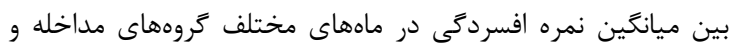

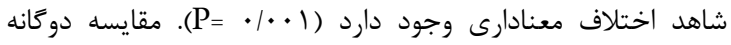

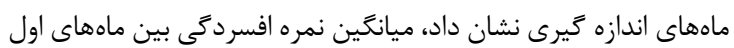

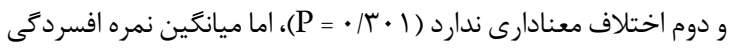

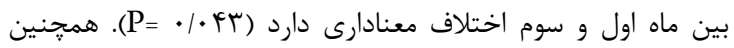

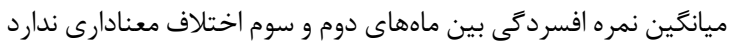

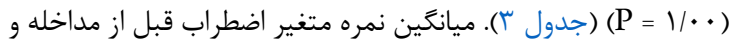

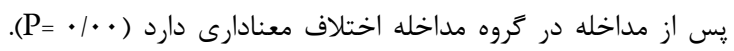

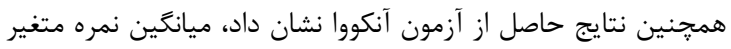

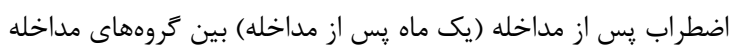

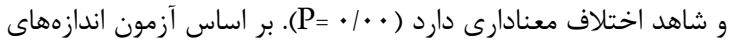

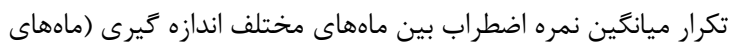


جدول rا. ميانكَين و انحراف معيار نمره متغيرها يّ از مداخله و در زمانهاى مختلف اندازه كيرى كروههاى مداخله و شاهد

\begin{tabular}{|c|c|c|c|c|c|c|}
\hline \multicolumn{2}{|c|}{ ماه سوم (ييخيرى) } & \multicolumn{2}{|c|}{ ماه دوم (ييگيرى) } & \multicolumn{2}{|c|}{ ماه اول (يس از مداخله) } & \multirow{2}{*}{ 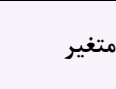 } \\
\hline انحراف معيار & ميانغين & انحراف معيار & ميانغين & انحراف معيار & ميانكين & \\
\hline & & & & & & استرس \\
\hline$r / 9$. & $V / \Delta \Lambda$ & F/qF & V/V & $\Delta / T T$ & $9 / \cdot \Delta$ & مداخله \\
\hline \multirow[t]{2}{*}{ Q/4T } & $\mid V / F V$ & V/AV & IV/re & N/Ar & $19 / 4 T$ & شاهد \\
\hline & & & & & & اضطراب \\
\hline $4 / 99$ & $4 / 11$ & $\Delta / r r$ & $f / r)$ & $\Delta / r V$ & F/ar & مداخله \\
\hline \multirow[t]{2}{*}{$N / \Delta F$} & $1 \cdot / \mathrm{VF}^{\mathrm{C}}$ & $V / 9 \Delta$ & $11 / 19$ & $V / q$. & 1.190 & شاهد \\
\hline & & & & & & افسردتى \\
\hline$F / \Delta q$ & $r / \wedge 9$ & $\Delta / r V$ & $r / r)$ & $\Delta / \Delta \Delta$ & $\Delta / \cdot \Delta$ & مداخله \\
\hline$N / 9 V$ & $\mid r / T r$ & $\Lambda / V V$ & $\mid r / 94$ & $9 / 4 q$ & $\mid r / 4 V$ & شاهد \\
\hline
\end{tabular}

بر استرس ادراى شده زنان مبتلا به يرفشارى خون، نشان داده است

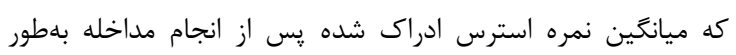

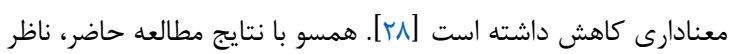

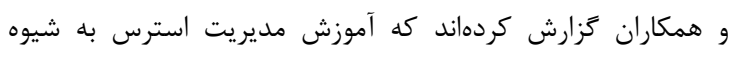

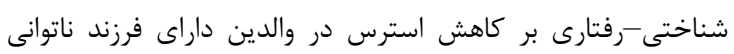

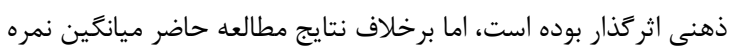

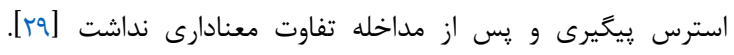

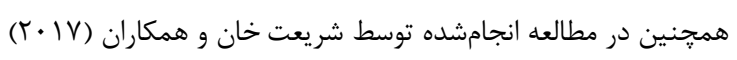

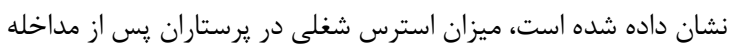

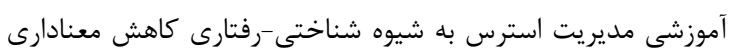

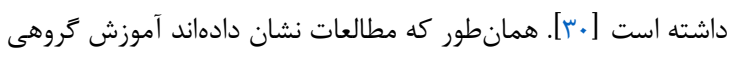

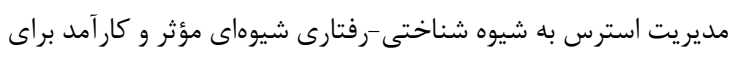

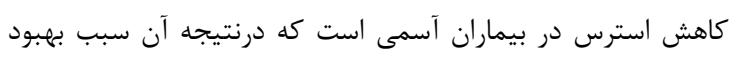
كيفيت زندكى بيماران مىشود.

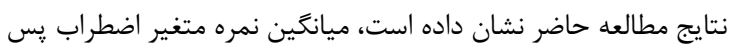

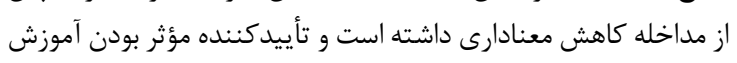

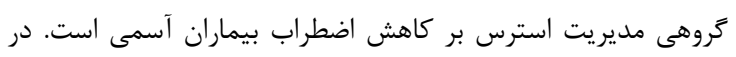

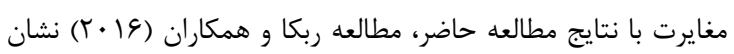

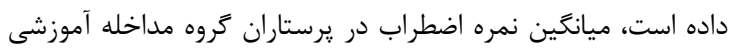

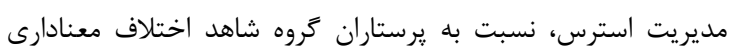

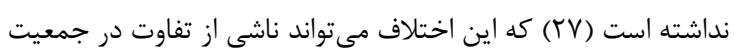

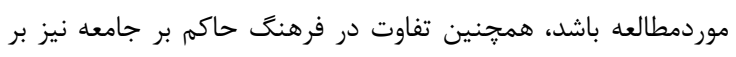

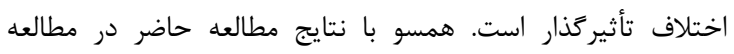

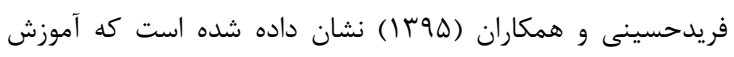

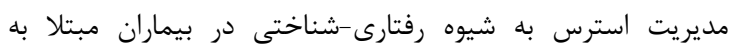

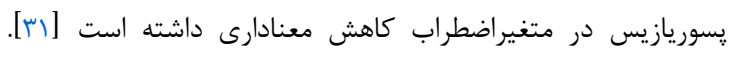

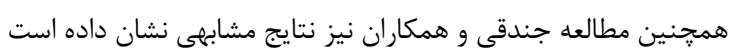

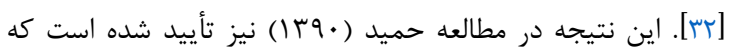

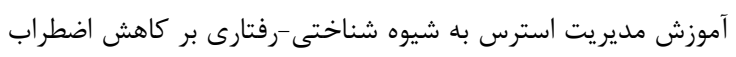

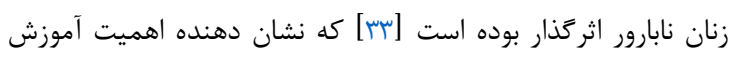

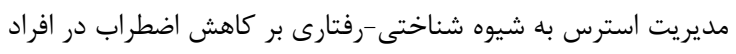
مبتلابه بيمارىهاى مزمن است.

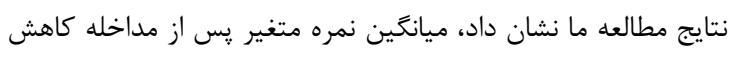

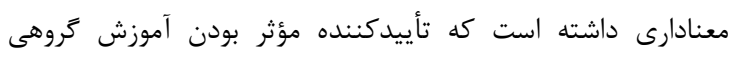

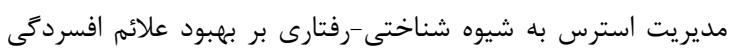

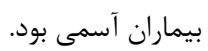

نتايج حاصل از آزمون آنووا نشان داد، افسردگى براى افراد كروه مداخله

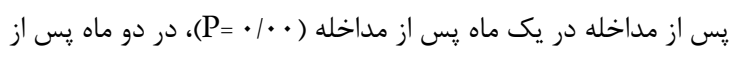

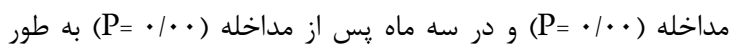

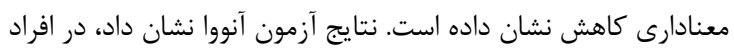

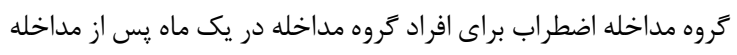

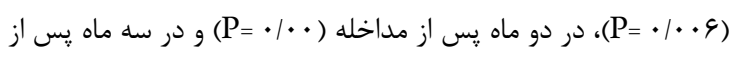

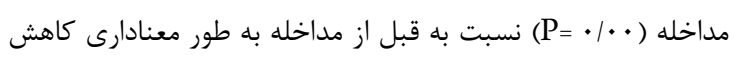

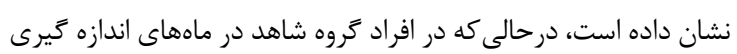
افزايش معنادارى نشان داده است.

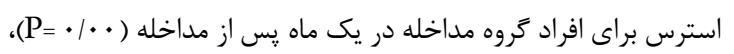

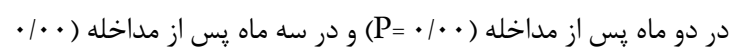

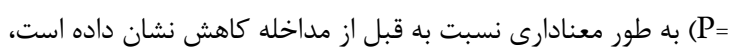

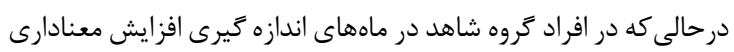
نشان داده است. نتايج آزمون تى مستقل نشان داد، تفاضل ميانكَين نمره بسترى در افراد

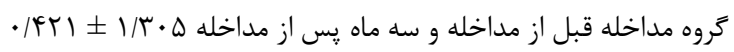

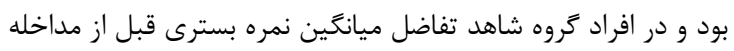

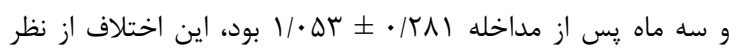

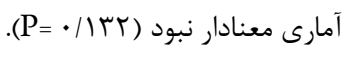

نتايج مطالعه حاضر نشان داده است كه آموزش گروهى مديريت استرس

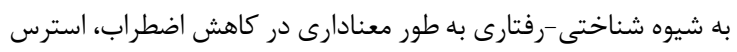

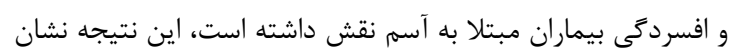

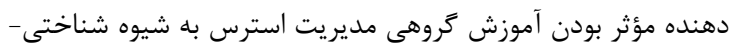

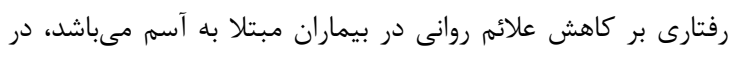

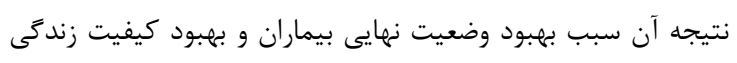

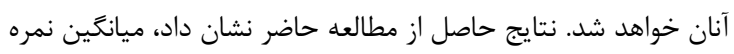

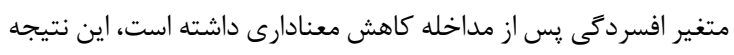

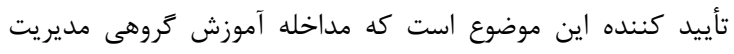

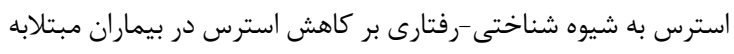

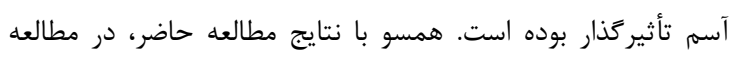

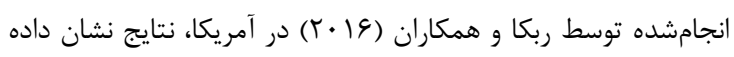

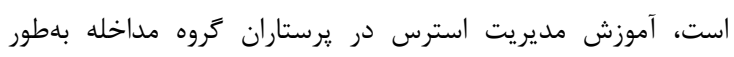

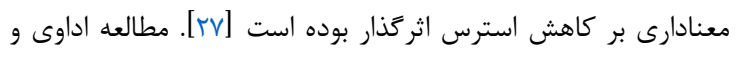

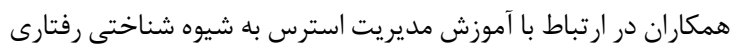


در آمريكا نشان داده است كه كودكان مبتلابه آسم كه مراقبتهاى

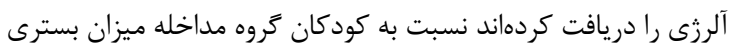

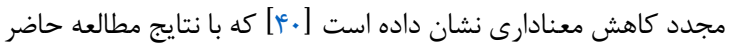

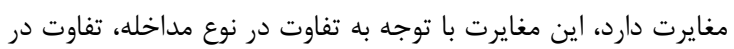

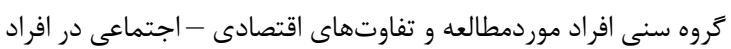
مورد مطالعه قابل توجيه است.

\section{نتيجه كيرى}

درمجموع يافتهاى اين يزوهش نشان داد آموزش گروهى مديريت

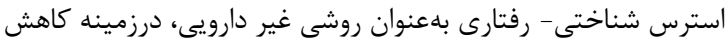

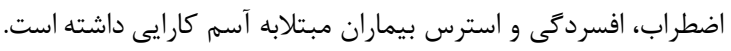

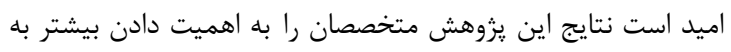

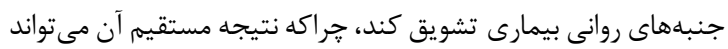

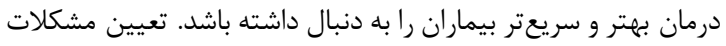

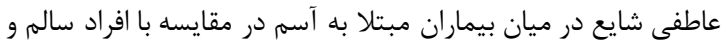

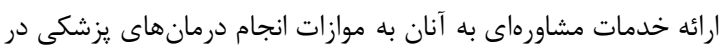

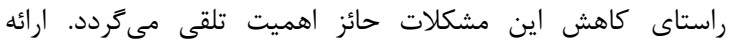

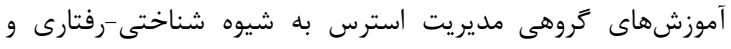

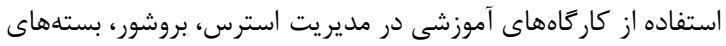

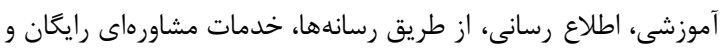

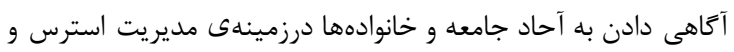

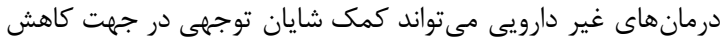

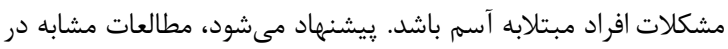

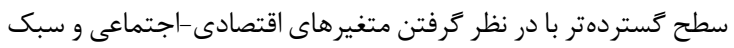
زندگى در بيماران مبتلابه بيمارىهاى مزمن انجام كَريرد.

ملاحظات اخلاقى

در اين مطالعه، نكات اخلاقى طبق :بروتكلهاى اخلاقى مطالعات

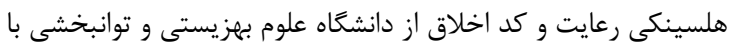

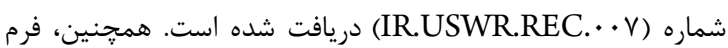
رضايتنامه شركت در يزوهش توسط همه آزمودنى ها امضا شد. شده الن.

حمايت مالى اين يزوهش با حمايت مالى دانشعاه علوم بهزيستى و توانبخشى انجام

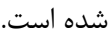

\section{سهم نويسندگان}

خانم فاطمه احمدى و آقاى مسعود فلاحى-خشكناب در ايده اوليه

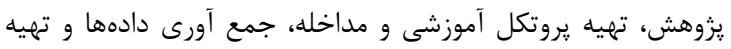

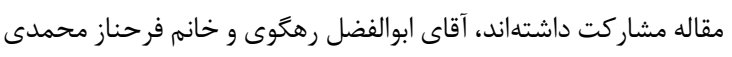

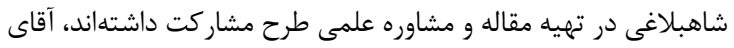

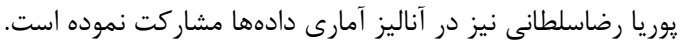

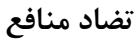

بدينوسيله نويسندكان اعلام مىنمايند كه هيجَّونه تعارض منافعى ندارد.
همسو با نتايج مطالعه ما، مطالعه استاگل و همكاران نشان داده است،

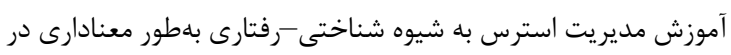

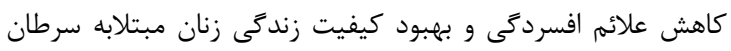

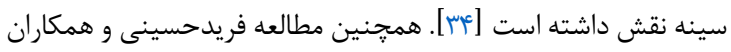

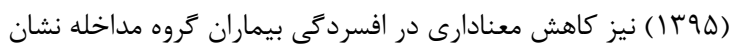

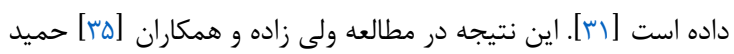

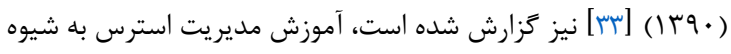

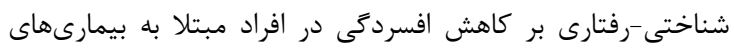

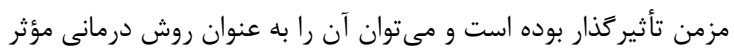
درمانى غير دارويى به كار خرفت.

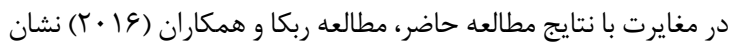

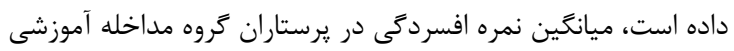

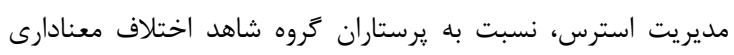

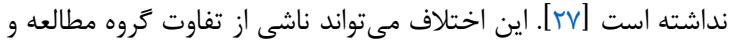

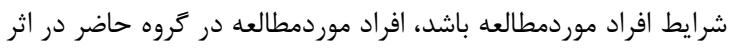
بيمارى مزمن آسم و شرايط و مشكلات ناشى از بيمارى تحت تأثير

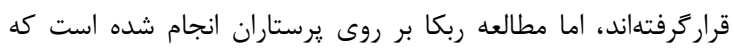

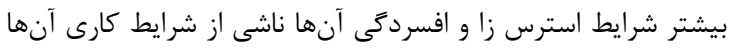
مىباشد كه مىتواند اين اختلاف را تبيين كند.

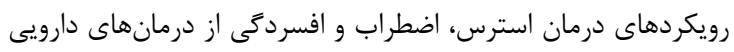

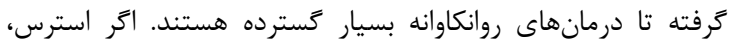

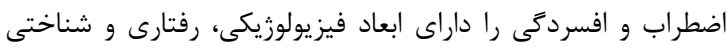

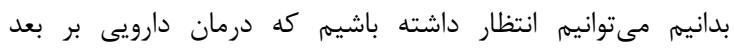

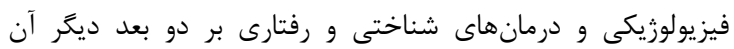
تأثير كذار هستند. نتايج مطالعه حاضر نشان داد، بين ميانگين دفعات بسترى قبل و وبعد

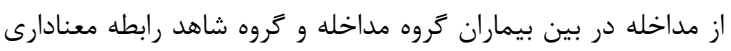

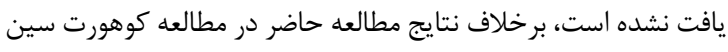

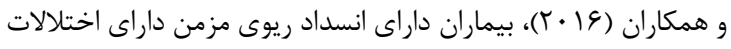

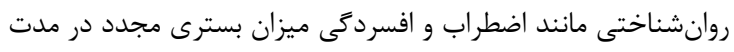

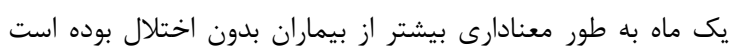

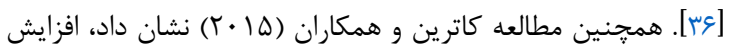

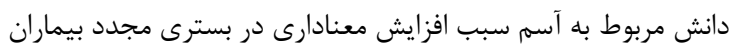

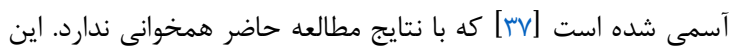

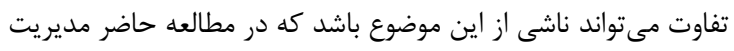

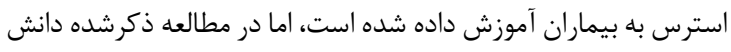

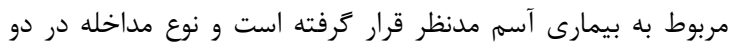

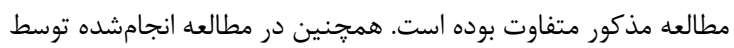

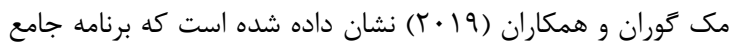

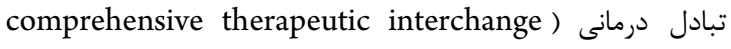
در بيماران مبتلابه ناراحتى مزمن ريوى منجر به كاهش (program

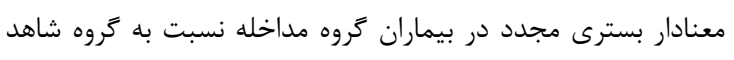

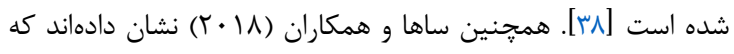

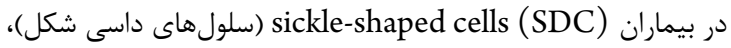
افسردى بلهطور معنادارى سبب افزايش بسترى مجدد در يكى ماه اول

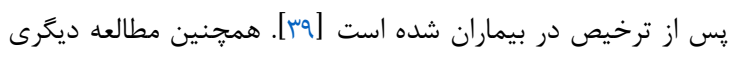




$$
\begin{aligned}
& \text { بهداشتى درمانى، در راستاى افزايش كيفيت زندگى بيماران مبتلا به }
\end{aligned}
$$

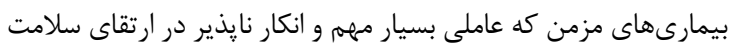

$$
\begin{aligned}
& \text { جامعه است كام بردارند. } \\
& \text { سياسگذارى }
\end{aligned}
$$$$
\text { اين مقاله حاصل از پايان نامه دانشجويى كارشناسى ارشد رشته }
$$

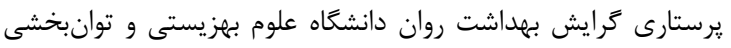

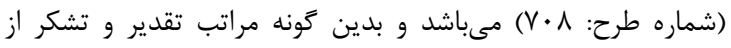

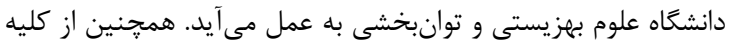

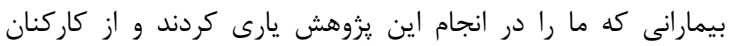

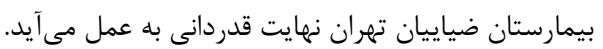

\section{References}

1. Holgate ST, Arshad HS, Roberts GC, Howarth PH, Thurner P, Davies DE. A new look at the pathogenesis of asthma. Clin Sci (Lond). 2009;118(7):439-50. doi: 10.1042/CS20090474 pmid: 20025610

2. Kasper D, Fauci A, Hauser S, Longo D, Jameson J, Loscalzo J. Harrison's principles of internal medicine: McGraw-Hill Professional Publishing; 2018.

3. Loerbroks A, Bosch JA, Sheikh A, Yamamoto S, Herr RM. Reports of wheezing and of diagnosed asthma are associated with impaired social functioning: Secondary analysis of the cross-sectional world health survey data. J Psychosom Res. 2018;105:52-7. doi: 10.1016/j.jpsychores.2017.12.008 pmid: 29332634

4. CgC-AoAahwcgadh AJ. 2019.

5. Arash M, Tajvidi M, Shogi M. Assessing effects of asthma on patients'functional status and life. J Urmia Nurs Midwife Facult. 2010;8(1):1-5.

6. CgC-A-MRADoAahwcgamrdhA.

7. Shalowitz MU, Berry CA, Quinn KA, Wolf RL. The relationship of life stressors and maternal depression to pediatric asthma morbidity in a subspecialty practice. Ambulator Pediatric. 2001;1(4):185-93. doi: $10.1367 / 1539$ -

4409(2001)001<0185:TROLSA > 2.0.CO;2

8. Scott KM, Von Korff M, Ormel J, Zhang MY, Bruffaerts R, Alonso J, et al. Mental disorders among adults with asthma: results from the World Mental Health Survey. Gen Hosp Psychiatry. 2007;29(2):123-33. doi: 10.1016/j.genhosppsych.2006.12.006 pmid: 17336661

9. Pietras T, Witusik A, Panek M, Hołub M, Gałecki P, Wujcik R. Anxiety and depression in patients with obstructive diseases. Polski Merkuriusz Lekarski: Organ Polskiego Towarzystwa Lekarskiego. 2009;26(156):6315.

10. Rosenblatt MS. Association between shorter length of stay (LOS) and readmission for complication exists. Ann Surg. 1996;224(2):236-8. doi: 10.1097/00000658199608000-00024 pmid: 8757390

11. Ritz T, Steptoe A, DeWilde S, Costa M. Emotions and stress increase respiratory resistance in asthma.

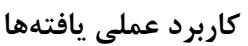

$$
\begin{aligned}
& \text { از آنجا كه آموزش مديريت استرس به شيوه شناختى-رفتارى بر كاهش }
\end{aligned}
$$

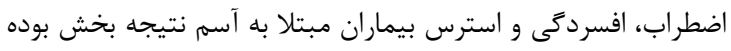

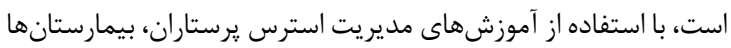

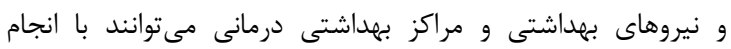

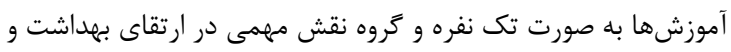

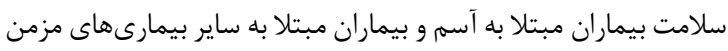

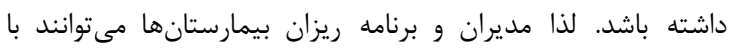

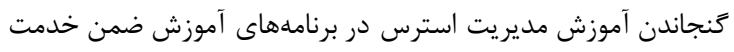

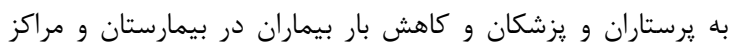

Psychosom Med. 2000;62(3):401-12. doi: 10.1097/00006842-200005000-00014 pmid: 10845354

12. Bray MA, Theodore LA, Patwa SS, Margiano SG, Alric JM, Peck HL. Written emotional expression as an intervention for asthma. Psychol School. 2003;40(2):193-207. doi: 10.1002/pits. 10078

13. Cohen BM. Psychological factors in asthma: A review of their aetiological and therapeutic significance. Postgrad Med J. 1971;47(550):533. doi: 10.1136/pgmj.47.550.533 pmid: 4929396

14. Hampel P, Rudolph H, Stachow R, Petermann F. Multimodal patient education program with stress management for childhood and adolescent asthma. Patient Educat Counsel. 2003;49(1):59-66. doi: 10.1016/S0738-3991(02)00046-0

15. Tousman S, Zeitz H, Bristol C, Taylor L. A pilot study on a cognitive-behavioral asthma self-management program for adults. Chron Respir Dis. 2006;3(2):73-82. doi: 10.1191/1479972306cd103oa pmid: 16729765

16. Jabalameli S, Moulavi H. Efficacy of cognitive-behavioral stress management intervention on quality of life and blood pressure in female patients with hypertension. Sci J Kurdistan Univ Med Sci. 2010;15(2):88-97.

17. Linden DVD, Keijsers GP, Eling P, Schaijk RV. Work stress and attentional difficulties: An initial study on burnout and cognitive failures. Work Stress. 2005;19(1):23-36. doi: 10.1080/02678370500065275

18. Norte CE, Souza GGL, Pedrozo AL, Mendonça-de-Souza ACF, Figueira I, Volchan E. Impact of cognitive-behavior therapy on resilience-related neurobiological factors. Arch Clinic Psychiatr (São Paulo). 2011;38(1):43-5. doi: 10.1590/S0101-60832011000100009

19. Emami M, Askarizade G, Faziltpour M. Effectiveness of cognitive-behavioral stress management group therapy on resilience and hope in women with breast cancer. 2017.

20. Tabibzadeh F, Azar FS. The effect of cognitive-behavioral therapy on the resilience and obsessive rumination among multiple sclerosis (MS) patients. 2016.

21. Charmchi N, Asgari P, Hafezi F, Makvandi B, Bakhtiyarpour S. The effect of "cngitive behavior therapy" 
in method group on anxiety and psychological resilience of menopausal women.2016.

22. Asemi Zavareh AC KA N, Mehrabi HA, Jahangazi A. The effectiveness of cognitive-behavioral stress management on depression, anxiety and stress in female patients with psychotic vertigo. Behav Sci Res. 2011;11(5):352-62.

23. Lovibond PF, Lovibond SH. The structure of negative emotional states: Comparison of the depression anxiety stress scales (DASS) with the beck depression and anxiety inventories. Behav Res Therap. 1995;33(3):335-43. doi: 10.1016/0005-7967(94)00075-U

24. Antony MM, Bieling PJ, Cox BJ, Enns MW, Swinson RP. Psychometric properties of the 42-item and 21-item versions of the depression anxiety stress scales in clinical groups and a community sample. Psychologic Assessment. 1998;10(2):176. doi: 10.1037/10403590.10.2.176

25. Asghari MM, Saed F, Dibajnia P, Zangeneh J. A preliminary validation of the depression, anxiety and stress scales (dass) in non-clinical sample.2008.

26. Moradipanah F. The effect of music on anxiety stress and mild depression patients undergoing cardiac catheterization [dissertation]. Tehran: TarbiyatModarres University; 2005.

27. Hersch RK, Cook RF, Deitz DK, Kaplan S, Hughes D, Friesen MA, et al. Reducing nurses' stress: A randomized controlled trial of a web-based stress management program for nurses. Appl Nurs Res. 2016;32:18-25. doi: 10.1016/j.apnr.2016.04.003 pmid: 27969025

28. Adavi A, Marghmalaki RF, Madmoli Y. The effect of cognitive-behavioral stress management training on perceived stress in females with hypertension. Iran Nurs Sci Associat. 2017;6(1):56-61. doi: 10.21859/ijnr-11052

29. Nazer M, Riyahi N, Mokhtaree M. The effect of stress management training with cognitive behavioral style on stress and mental health of parents of children with intellectual disabilities. Arch Rehabilit. 2016;17(1):32-41. doi: $10.20286 /$ jrehab-170130

30. Shariatkhah J, Farajzadeh Z, Khazaee K. The effects of cognitive-behavioral stress management on nurses' job stress. Iran J Nurs Midwife Res. 2017;22(5):398.

31. Faridhosseini F. Effectiveness of cognitive-behavioral stress management on anxiety, depression and quality of life in patients with psoriasis. Med J Mashhad Univ Med Sci. 2016;59(5):337-44.
32. Jandaghi F, Neshat DH, Kalantari M, Jabalameli S. The effectiveness of cognitive-behavioral stress management group training on anxiety and depression of addicts under methadone maintenance therapy $(\mathrm{mmt})$. Clinic Psychol. 2013;4(4):41-50.

33. Hamid N. The effectiveness of stress management on depression, anxiety and fertilization of infertile women. (Persian). J Behav Sci. 2011;5(1):55-60.

34. Stagl JM, Bouchard LC, Lechner SC, Blomberg BB, Gudenkauf LM, Jutagir DR, et al. Long-term psychological benefits of cognitive-behavioral stress management for women with breast cancer: 11-year follow-up of a randomized controlled trial. Cancer. 2015;121(11):1873-81. doi: 10.1002/cncr.29076 pmid: 25809235

35. Valizadeh R, Sohrabnejad S, Mehraban S, Ahmadboukani S. Effectiveness of cognitive-behavioral based stress management training on depression of MS female patients. Sci J Ilam Univ Med Sci. 2015;23(5):91-8.

36. Singh G, Zhang W, Kuo YF, Sharma G. Association of psychological disorders with 30-day readmission rates in patients with COPD. Chest. 2016;149(4):905-15. doi: 10.1378/chest.15-0449 pmid: 26204260

37. Auger KA, Kahn RS, Davis MM, Simmons JM. Pediatric asthma readmission: asthma knowledge is not enough? J Pediatr. 2015;166(1):101-8. doi: 10.1016/j.jpeds.2014.07.046 pmid: 25241184

38. McGurran MA, Richter LM, Leedahl ND, Leedahl DD. Impact of a comprehensive COPD therapeutic interchange program on 30-day readmission rates in hospitalized patients. Pharmacy Therapeutic. 2019;44(4):185.

39. Saha A, Poojary P, Pandya D, Kumar A, Nadkarni G, Kaur S. Association of 30 day readmission between psychosocial, behavioral and epidemiological factors in adult SCD patients. Am Soc Hematol. 2018. doi: 10.1182/blood-2018-99-117756

40. Ruffner MA, Henrickson SE, Chilutti M, Grundmeier R, Spergel JM, Brown-Whitehorn TF. Improving allergy office scheduling increases patient follow up and reduces asthma readmission after pediatric asthma hospitalization. Ann Allergy Asthma Immunol. 2018;121(5):561-7. doi: 10.1016/j.anai.2018.08.015 pmid: 30170026 